\title{
MORTALITY RATES FROM CANCER AND TUBERCULOSIS
}

\author{
BY \\ J. L. HAYBITTLE \\ From the Radiotherapeutic Centre, Addenbrooke's Hospital, Cambridge
}

The relationship between cancer and tuberculosis mortality rates was first noted by Coghlan (1903), and investigated in great detail by Cherry in a series of papers and books published between 1924 and 1945. Cherry's original work was confirmed and extended by Cruickshank (1939), and more recently Paxon (1956) and Campbell (1961a, b) have reported further work on the subject.

The factual findings of all these authors may be summarized as follows:

(i) In England and Wales the combined crude mortality rate for tuberculosis and cancer has remained approximately constant since 1893 . Similar constancy has also been demonstrated in the Australian mortality rates.

(ii) In groups of people following the same occupation or living in the same area, the mortality from tuberculosis and cancer usually runs parallel, i.e. a higher cancer mortality rate is associated with a higher tuberculosis rate. This is in contrast to $(i)$, where over a period of time in the same area a falling tuberculosis rate has been associated with a rising cancer rate.

(iii) For each of the five England and Wales cohorts who were aged 15-25 years in 1841, $1851,1861,1871$, and 1881 respectively, the ratio of deaths from cancer and tuberculosis to deaths from all causes above the age of 15 years was almost exactly $0 \cdot 23$.

These findings have been used by Cherry, Cruickshank, and Paxon to support the suggestion that there is a causal relationship between cancer and tuberculosis. Campbell (1961a), however, has criticized this suggestion on the grounds that the relationships have been based upon "crude" and not age-standardized mortality rates, and he points out that if the age-structure of the population "had not changed, the joint tuberculosis and cancer mortality rate would not have remained constant". This criticism, although valid, cannot be applied to the cohort analysis used by Cherry (1925), and the fact that the proportion of each cohort ultimately dying of either cancer or tuberculosis has remained remarkably constant seems unlikely to be an artefact produced entirely by the interaction of the factors mentioned by Campbell, i.e. improving social conditions, improving health services, and more urbanization, industrialization, and tobacco smoking. Although these factors might interact in such a way as to cause a decrease in the tuberculosis rate and an increase in the cancer rate to occur concurrently, the fact that the fall in the probability that an individual will ultimately die of tuberculosis may have been almost exactly compensated for by the increase in the probability that he will ultimately die of cancer is an unexpected finding.

It would be of particular interest to extend Cherry's cohort analysis to later cohorts in whom more recent changes in cancer mortality such as the increase in deaths from lung cancer might be taking effect. Unfortunately the data required for this extension is not yet available, but use may be made of an alternative approach which has been described in a previous paper (Haybittle, 1962). The method is to study mortality trends in the birth-standardized populations of England and Wales, i.e. the populations that would have existed at any time if the number of live births per year had remained constant. It has been shown (Haybittle, 1962) that the ratio, $R^{\prime}$, of deaths from cancer to deaths from all causes in the birth-standardized populations can be used to show trends in the probability, $R$, that an individual will ultimately die of cancer. Furthermore, trends in $R$ may be observed by this means 
before the full information is available for cohort analysis. The cancer and tuberculosis mortality rates in the birth-standardized populations of England and Wales have therefore been calculated for quinquennia from 1921 to 1955 and for the 4-year period 1956-59. The results are described in the next section.

Mortality Rates IN THE BiRTh-STANDARDIZED POPULATIONS

Cancer age-specific mortality rates were taken from "Cancer Statistics in England and Wales, 1901-1955" (General Register Office, 1957), the pre1939 rates being corrected for the change in the method of registration that took place in 1939. The rates for 1956-59 were calculated from the figures published in the Registrar General's "Statistical Review" for those years. Deaths from Hodgkin's disease and leukaemia were not included.

Tuberculosis rates were calculated using the figures published in the Statistical Reviews for "Tuberculosis-All Forms", and the aggregate midyear populations given in "Cancer Statistics in England and Wales, 1901-55" (1957). Again, pre-1939 rates were corrected for the 1939 change in registration procedure. The birth-standardized populations to which these age-specific mortality rates were applied, have been tabulated in a previous paper (Haybittle, 1962).

For comparison, age-specific rates were similarly calculated for deaths during the same period from "Pneumonia-All Forms", and these rates were also applied to the birth-standardized populations. Pneumonia was chosen as being another disease similar to tuberculosis in that its contribution to the general mortality has decreased markedly during the last $\mathbf{4 0}$ years.

Fig. 1 and Table I (opposite) show the variation in the mortality rate in the birth-standardized populations (designated the birth-standardized rate or B.S.R.) for cancer, tuberculosis, and pneumonia separately, and also for cancer and tuberculosis combined, and for cancer and pneumonia combined. It may be seen that the constancy of the combined cancer and tuberculosis rate which showed itself in the crude mortality rate data, is no longer apparent when the effect of birth rate changes has been eliminated. There has in fact been a continuous fall in the B.S.R. for both males and females during the period studied. Up to 1951, the B.S.R. for pneumonia followed that for tuberculosis closely, but during the last decade the fall in the pneumonia rate has ceased and as a result the combined cancer and pneumonia B.S.R. has begun to rise.
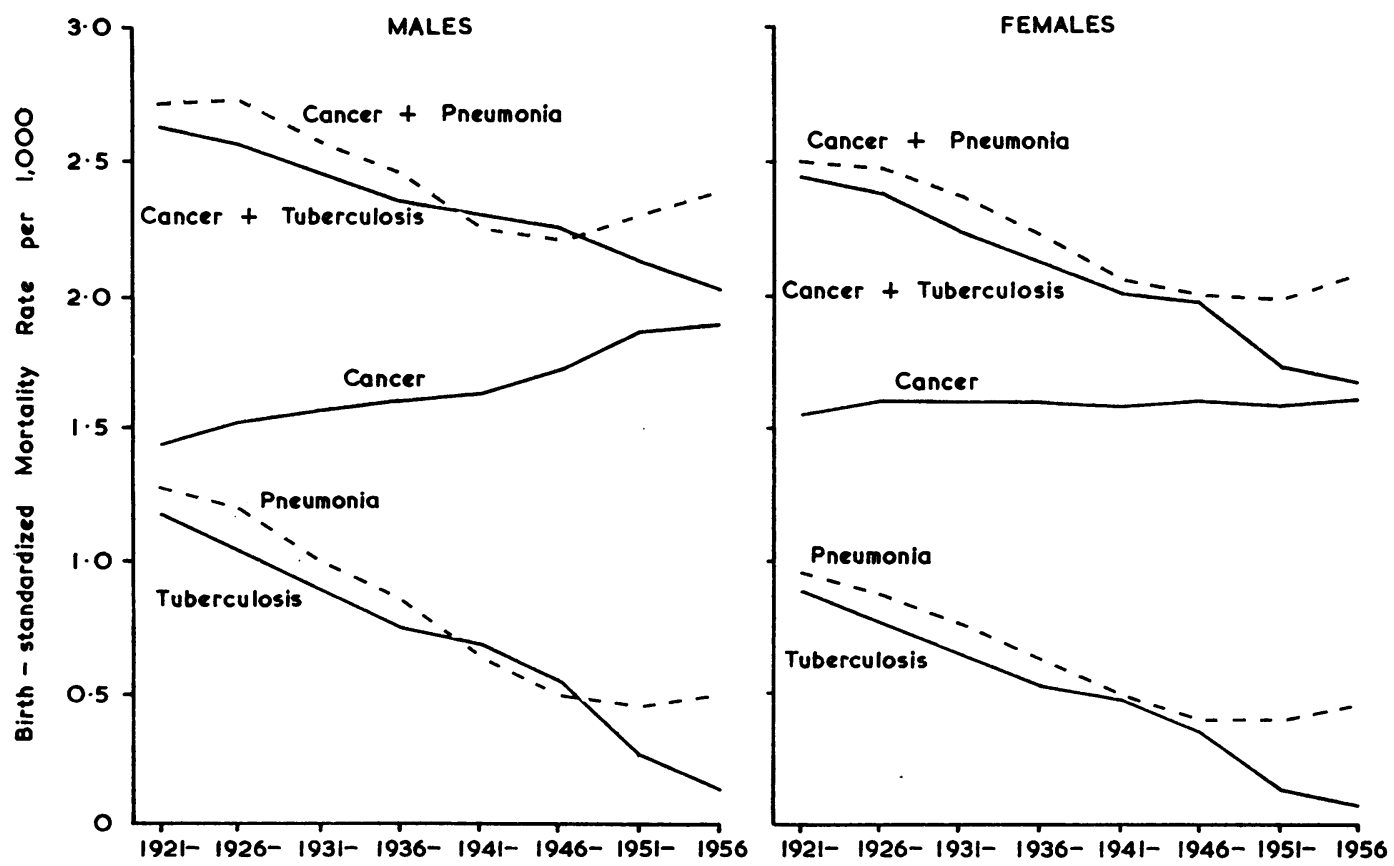

FIG. 1.-Birth-standardized mortality rates per 1,000 in England and Wales averaged over 5-year periods, by sex. 
TABLE I

MORTALITY RATES PER 1,000 IN THE BIRTH-STANDARDIZED POPULATIONS OF ENGLAND AND WALES, 1921-59, BY SEX

\begin{tabular}{|c|c|c|c|c|c|c|c|c|c|c|c|c|}
\hline \multirow{2}{*}{\multicolumn{4}{|c|}{ Cause of Death }} & \multirow{2}{*}{ Sex } & \multicolumn{8}{|c|}{ Year } \\
\hline & & & & & $1921-$ & $1926-$ & $1931-$ & 1936 & $1941-$ & $1946-$ & $1951-$ & 1956-59 \\
\hline \multirow{2}{*}{ Cancer } & \multirow{2}{*}{$\cdots$} & \multirow{2}{*}{. } & \multirow{2}{*}{$\cdots$} & $\mathbf{M}$ & $1 \cdot 44$ & $1 \cdot 53$ & $1 \cdot 57$ & $1 \cdot 61$ & $1 \cdot 62$ & $1 \cdot 73$ & $1 \cdot 85$ & $1 \cdot 89$ \\
\hline & & & & $\mathbf{F}$ & $1 \cdot 55$ & 1.61 & $1 \cdot 60$ & 1.61 & $1 \cdot 58$ & $1 \cdot 61$ & $1 \cdot 59$ & $1 \cdot 60$ \\
\hline \multirow{2}{*}{ Tuberculosis } & \multirow{2}{*}{. } & \multirow{2}{*}{$\cdots$} & \multirow{2}{*}{$\cdots$} & $\mathbf{M}$ & $1 \cdot 19$ & $1 \cdot 04$ & 0.89 & 0.74 & 0.67 & 0.53 & $0 \cdot 27$ & $0 \cdot 13$ \\
\hline & & & & $\mathbf{F}$ & 0.89 & 0.77 & 0.64 & 0.52 & 0.48 & $0 \cdot 37$ & $0 \cdot 13$ & 0.05 \\
\hline \multirow{2}{*}{ Pneumonia } & \multirow{2}{*}{. } & \multirow{2}{*}{. } & \multirow{2}{*}{. } & $\mathbf{M}$ & $1 \cdot 29$ & $1 \cdot 21$ & $1 \cdot 00$ & 0.86 & 0.63 & 0.48 & 0.46 & 0.49 \\
\hline & & & & $F$ & 0.95 & 0.87 & $0 \cdot 76$ & 0.63 & 0.49 & 0.40 & $0 \cdot 40$ & 0.45 \\
\hline \multirow{2}{*}{\multicolumn{3}{|c|}{ Cancer + Tuberculosis . }} & \multirow{2}{*}{$\cdots$} & $\mathbf{M}$ & $2 \cdot 63$ & $2 \cdot 57$ & $2 \cdot 46$ & $2 \cdot 35$ & $2 \cdot 29$ & $2 \cdot 26$ & $2 \cdot 12$ & $2 \cdot 02$ \\
\hline & & & & $\mathbf{F}$ & $2 \cdot 44$ & $2 \cdot 38$ & $2 \cdot 24$ & $2 \cdot 13$ & $2 \cdot 06$ & $1 \cdot 98$ & $1 \cdot 79$ & $1 \cdot 65$ \\
\hline \multirow{2}{*}{\multicolumn{2}{|c|}{ Cancer + Pneumonia }} & \multirow{2}{*}{. } & \multirow{2}{*}{$\ldots$} & $\mathbf{M}$ & $2 \cdot 73$ & $2 \cdot 74$ & $2 \cdot 57$ & $2 \cdot 47$ & $2 \cdot 25$ & $2 \cdot 21$ & $2 \cdot 31$ & $2 \cdot 38$ \\
\hline & & & & $\mathbf{F}$ & $2 \cdot 50$ & $2 \cdot 48$ & $2 \cdot 36$ & $2 \cdot 24$ & $2 \cdot 07$ & 2.01 & 1.99 & 2.05 \\
\hline \multirow{2}{*}{ All Causes } & \multirow{2}{*}{$\cdots$} & & \multirow{2}{*}{$\cdots$} & $\mathbf{M}$ & $15 \cdot 35$ & $14 \cdot 90$ & $14 \cdot 10$ & $13 \cdot 92$ & $12 \cdot 70$ & $11 \cdot 50$ & $11 \cdot 33$ & $10 \cdot 88$ \\
\hline & & & & $\mathbf{F}$ & $13 \cdot 97$ & $13 \cdot 52$ & $12 \cdot 90$ & $12 \cdot 50$ & $11 \cdot 11$ & $10 \cdot 48$ & $9 \cdot 98$ & $9 \cdot 64$ \\
\hline
\end{tabular}

In Fig. 2, the ratio, $R^{\prime}$, for cancer is plotted, and also the similar ratio to all causes of death for cancer and tuberculosis combined, and for cancer and pneumonia combined.

The values are tabulated in Table II (overleaf). It may be seen that in females, apart from a rise in 1941-50, the ratio for cancer and tuberculosis combined has remained practically constant at a value of about $0 \cdot 175$. This is in contrast to the cancer and pneumonia ratio which has continued to rise since 1941 .

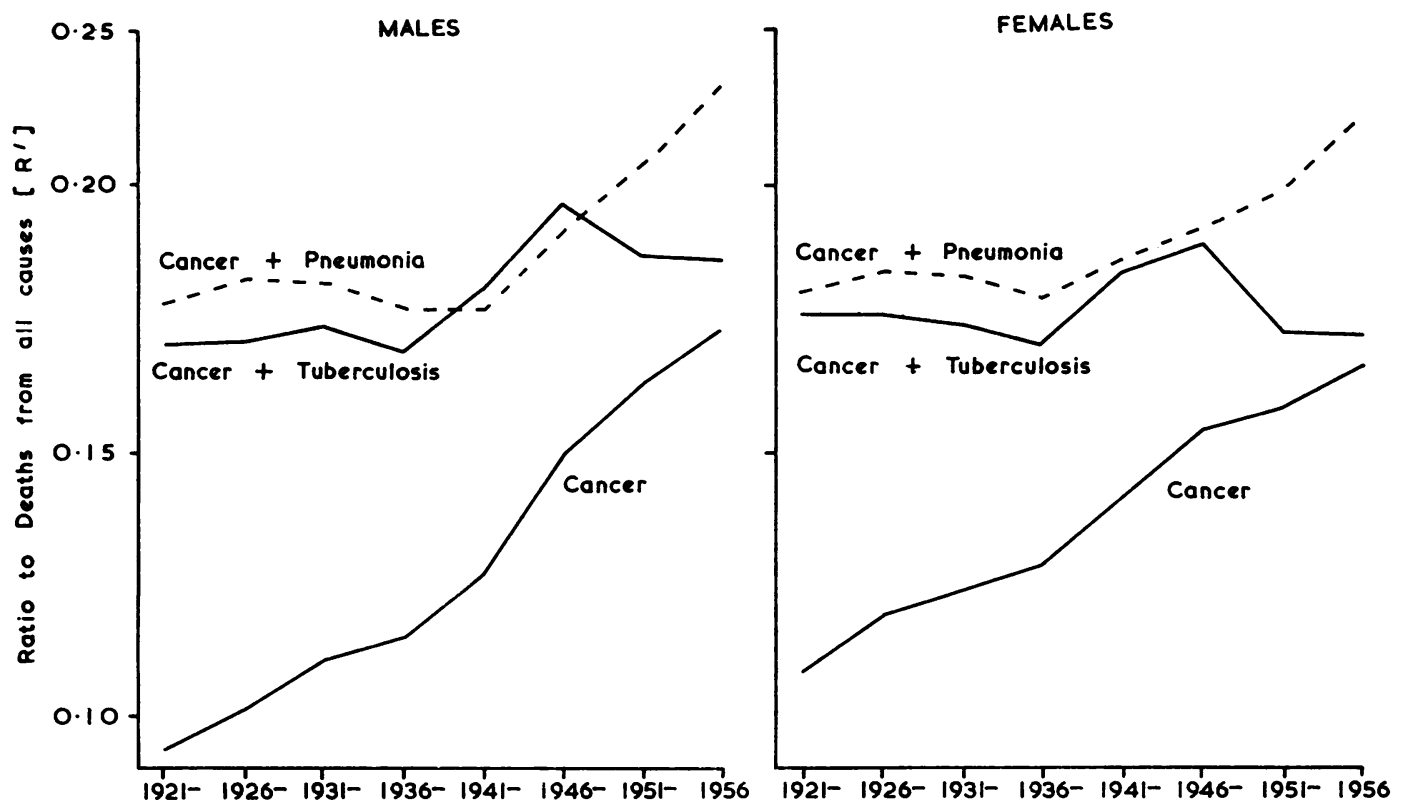

Fic. 2.-Deaths from specified causes as a fraction of deaths from all causes in the birth-standardized populations of England and Wales averaged over 5-year periods, by sex. 
TABLE II

DEATHS FROM SPECIFIED CAUSES AS A FRACTION OF DEATHS FROM ALL CAUSES IN THE BIRTH-STANDARDIZED POPULATIONS OF ENGLAND AND WALES, 1921-59, BY SEX

\begin{tabular}{|c|c|c|c|c|c|c|c|c|c|c|c|c|c|}
\hline \multirow{2}{*}{\multicolumn{5}{|c|}{ Cause of Death }} & \multirow{2}{*}{ Sex } & \multicolumn{8}{|c|}{ Year } \\
\hline & & & & & & 1921- & $1926-$ & 1931- & 1936 & 1941- & $1946-$ & 1951- & $1956-59$ \\
\hline \multirow{2}{*}{ Cancer } & \multirow{2}{*}{$\cdots$} & \multirow{2}{*}{$\cdots$} & \multirow[b]{2}{*}{$\cdots$} & \multirow{2}{*}{$\cdots$} & $\mathbf{M}$ & $0 \cdot 094$ & $0 \cdot 103$ & $0 \cdot 111$ & $0 \cdot 116$ & $0 \cdot 128$ & $0 \cdot 150$ & $0 \cdot 167$ & $0 \cdot 174$ \\
\hline & & & & & $\mathbf{F}$ & $0 \cdot 111$ & $0 \cdot 119$ & $0 \cdot 124$ & $0 \cdot 129$ & $0 \cdot 142$ & $0 \cdot 154$ & $0 \cdot 159$ & $0 \cdot 166$ \\
\hline \multirow{2}{*}{\multicolumn{2}{|c|}{ Tuberculosis }} & \multirow{2}{*}{. } & \multirow{2}{*}{$\cdots$} & \multirow{2}{*}{. } & $\mathbf{M}$ & 0.078 & 0.070 & 0.063 & 0.053 & 0.053 & 0.046 & 0.024 & 0.012 \\
\hline & & & & & $\mathbf{F}$ & 0.064 & 0.057 & 0.050 & 0.042 & 0.043 & 0.035 & $0 \cdot 013$ & 0.005 \\
\hline \multirow{2}{*}{\multicolumn{2}{|c|}{ Pneumonia }} & \multirow{2}{*}{$\cdots$} & \multirow{2}{*}{$\cdots$} & \multirow{2}{*}{. } & $\mathbf{M}$ & 0.084 & 0.081 & $0 \cdot 071$ & 0.062 & 0.050 & 0.042 & 0.041 & 0.045 \\
\hline & & & & & $\mathbf{F}$ & $0 \cdot 068$ & 0.064 & 0.059 & 0.050 & 0.044 & 0.038 & 0.040 & 0.047 \\
\hline \multirow{2}{*}{\multicolumn{4}{|c|}{ Cancer + Tuberculosis }} & \multirow{2}{*}{$\cdots$} & $\mathbf{M}$ & $0 \cdot 171$ & $0 \cdot 172$ & $0 \cdot 174$ & $0 \cdot 169$ & $0 \cdot 180$ & $0 \cdot 196$ & $0 \cdot 187$ & $0 \cdot 186$ \\
\hline & & & & & $\mathbf{F}$ & $0 \cdot 175$ & $0 \cdot 176$ & $0 \cdot 174$ & $0 \cdot 171$ & $0 \cdot 185$ & $0 \cdot 189$ & $0 \cdot 172$ & $0 \cdot 171$ \\
\hline \multirow{2}{*}{\multicolumn{3}{|c|}{ Cancer + Pneumonia }} & \multirow{2}{*}{$\cdots$} & \multirow{2}{*}{$\cdots$} & $\mathbf{M}$ & $0 \cdot 178$ & $0 \cdot 184$ & $0 \cdot 182$ & $0 \cdot 177$ & $0 \cdot 177$ & $0 \cdot 192$ & $0 \cdot 204$ & $0 \cdot 219$ \\
\hline & & & & & $\mathbf{F}$ & $0 \cdot 179$ & $0 \cdot 183$ & $0 \cdot 183$ & $0 \cdot 179$ & $0 \cdot 186$ & $0 \cdot 192$ & $0 \cdot 199$ & $0 \cdot 213$ \\
\hline
\end{tabular}

In males the rise in cancer and pneumonia combined is similar, but the combined cancer and tuberculosis ratio reached a peak in 1946-50, and has now fallen again, although not so far as the female ratio. As a result, the 1956-59 value is 0.186 compared with the 1921-25 value of $0 \cdot 171$, and there has therefore been a small increase in the combined ratio over the past 40 years.

Fig. 3 and Table III (opposite) show that the reduction in the death rate from tuberculosis has not been accompanied by a reduction in the average age at which deaths occur from cancer. In fact the mean age at death of cancer patients has increased very slightly over the period studied, by about $1 \frac{1}{2}$ and $2 \frac{1}{2}$ years in males and females respectively. The marked increase that has occurred since 1941 in the mean age at death of tuberculosis patients is also shown in Fig. 3. When the patients who die of either cancer or tuberculosis are considered as one group, their mean age at death is seen to have increased by 14 years between 1921 and 1959.

\section{Discussion}

In the previous paper (Haybittle, 1962), to which reference has already been made, it was shown that changes in the probability that an individual will ultimately die from a particular disease manifest themselves in the ratio of deaths from that disease
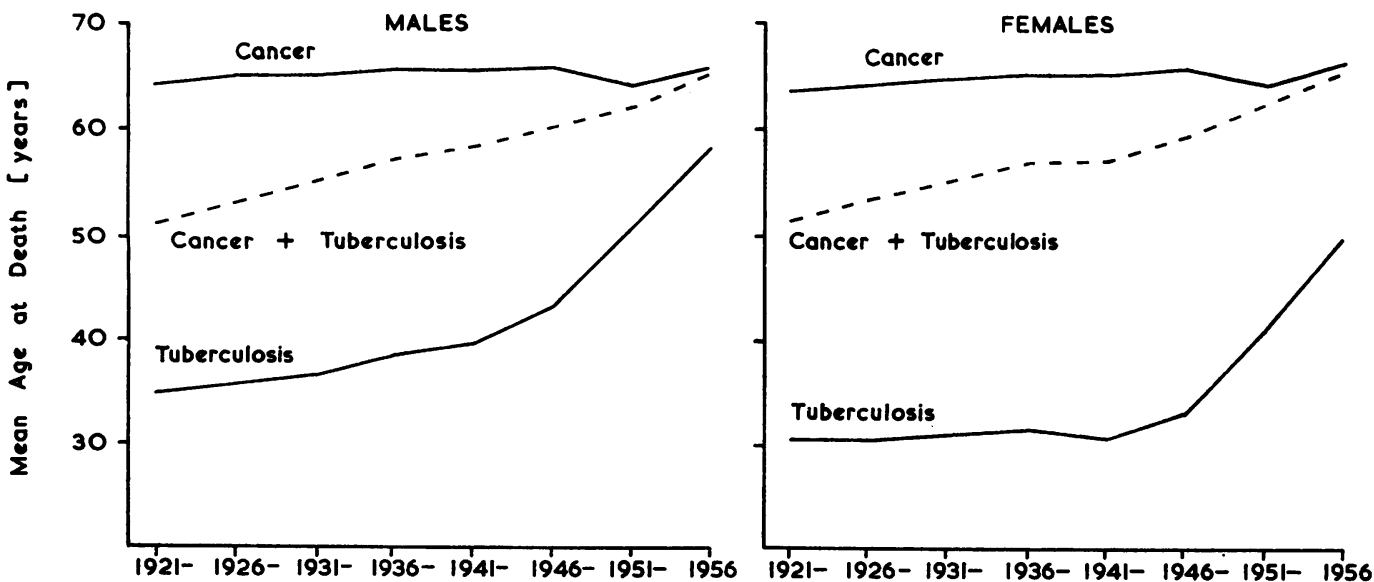

FIG. 3.-Mean age (yrs) at death from specified causes in the birth-standardized populations of England and Wales averaged over 5-year periods, by sex. 
TABLE III

MEAN AGE (YRS) AT DEATH FROM SPECIFIED CAUSES IN THE BIRTH-STANDARDIZED POPULATIONS OF ENGLAND AND WALES, 1921-59, BY SEX

\begin{tabular}{|c|c|c|c|c|c|c|c|c|c|c|c|c|}
\hline \multirow{2}{*}{\multicolumn{4}{|c|}{ Cause of Death }} & \multirow{2}{*}{ Sex } & \multicolumn{8}{|c|}{ Year } \\
\hline & & & & & $1921-$ & 1926 & 1931- & 1936 & $1941-$ & $1946-$ & $1951-$ & $1956-59$ \\
\hline \multirow{2}{*}{ Cancer } & \multirow{2}{*}{$\cdots$} & \multirow{2}{*}{$\cdots$} & \multirow{2}{*}{$\cdots$} & $\mathbf{M}$ & $64 \cdot 3$ & $65 \cdot 0$ & $65 \cdot 2$ & $65 \cdot 4$ & $65 \cdot 4$ & $65 \cdot 6$ & $63 \cdot 8$ & $65 \cdot 7$ \\
\hline & & & & $\mathbf{F}$ & $63 \cdot 7$ & $64 \cdot 2$ & $64 \cdot 5$ & $65 \cdot 0$ & $65 \cdot 0$ & $65 \cdot 6$ & $66 \cdot 0$ & $66 \cdot 2$ \\
\hline \multirow{2}{*}{ Tuberculosis } & \multirow{2}{*}{$\cdots$} & \multirow{2}{*}{$\cdots$} & \multirow{2}{*}{$\cdots$} & $\mathbf{M}$ & $35 \cdot 1$ & $35 \cdot 8$ & $36 \cdot 7$ & $38 \cdot 6$ & $39 \cdot 5$ & $42 \cdot 8$ & $50 \cdot 7$ & $57 \cdot 8$ \\
\hline & & & & $\mathbf{F}$ & $30 \cdot 6$ & $30 \cdot 5$ & $30 \cdot 9$ & $31 \cdot 4$ & $30 \cdot 7$ & $32 \cdot 9$ & $40 \cdot 6$ & $49 \cdot 5$ \\
\hline \multirow{2}{*}{\multicolumn{3}{|c|}{ Cancer + Tuberculosis }} & \multirow{2}{*}{$\cdots$} & $\mathbf{M}$ & $51 \cdot 2$ & $53 \cdot 1$ & $55 \cdot 0$ & $57 \cdot 2$ & $57 \cdot 8$ & $60 \cdot 1$ & $62 \cdot 2$ & $65 \cdot 4$ \\
\hline & & & & $F$ & $51 \cdot 5$ & $53 \cdot 3$ & $55 \cdot 0$ & 56.9 & $57 \cdot 0$ & $59 \cdot 5$ & $64 \cdot 2$ & $65 \cdot 4$ \\
\hline
\end{tabular}

to deaths from all causes in the birth-standardized populations. For the type of change that has occurred in cancer mortality over recent years, the time-lag between the date of birth of the cohort in which the change takes place and the date at which the change becomes apparent in the birthstandardized population is probably about 30 to 40 years. If we assume that the same time-lag exists for combined cancer and tuberculosis mortality, then we may conclude from the results of the previous section, that no marked change has occurred in the probability that an individual will ultimately die from either cancer or tuberculosis in cohorts born up to about 1920. Combined with Cherry's findings, the constancy of this probability is therefore demonstrated for people born in England and Wales over a period of about 90 years, apart from a small recent increase for males. It should be noted that Cherry's figure of 0.23 (as calculated by Cruickshank, 1939) refers to deaths above the age of 15 years, and is consequently higher than the values of about 0.18 found in this study which refer to deaths at all ages. In fact, if deaths below the age of 15 years are added to Cherry's 1861 and 1871 census units, and the figures are corrected to include all forms of tuberculosis, then the combined deaths from tuberculosis and cancer in these units amount to about 0.187 of the deaths from all causes, in very reasonable agreement with the values obtained in the birth-standardized populations.

A satisfactory explanation of the constancy of this ratio has yet to be found, and the main purpose of this paper is to present the facts rather than to offer an explanation. Evidence for a specific causal relationship between tuberculosis and cancer is not strong, although Campbell (1961b) has shown that pulmonary tuberculosis probably has a causal effect on lung cancer in males through non-specific changes such as scarring or metaplasia of the bronchial mucosa. According to this argument a reduction in the tuberculosis death-rate increases the proportion of cured tuberculosis cases in the population, and the increased cancer mortality arises because of the greater liability to cancer of this proportion. Cherry, Cruickshank, and Paxon all tend to take this view.

On the other hand, Pearl (1929) offered the alternative explanation that tuberculosis inhibited the development of cancer through some protective effect. Thus, as the number of the population infected with tuberculosis decreases, there will be a corresponding decrease of protection from cancer leading to an increase in cancer mortality. In this connexion it is of interest to note that pthiocol which occurs in the tuberculosis bacillus was found by Mitchell and Simon-Reuss (1952) to be a mitotic inhibitor in tissue cultures. It is difficult, however, to reconcile the concept of tuberculosis protecting against cancer with the facts that in certain geographical areas and living conditions, a high tuberculosis death-rate is associated with a high cancer rate and vice versa.

A further possibility is that a genetically determined cancer proneness (Goodhart, 1959) is linked with a similar proneness to tuberculosis, so that in any population group there are certain individuals who, when exposed to infection from tuberculosis bacilli or to carcinogens, are more likely to succumb to their effects than is the rest of the population. Thus, as environmental factors causing tuberculosis are reduced, more of these cancer-prone individuals live to an age when cancer is likely to develop, and as a result there is a corresponding increase in cancer mortality, the combined deaths from tuberculosis and cancer being mainly accounted for by these individuals. The constant proportion of deaths from cancer and tuberculosis combined would then suggest that the predominant conditioning factor leading to death from cancer may be genetical.

The genetical factor in cancer has been discussed by Huxley (1958). If a common factor predisposes to 
tuberculosis and cancer, then one might expect tuberculosis and cancer patients to show some other constitutional similarities. So far the evidence on this point is scanty. Sheldon, Hartl, and McDermott (1949) published some somatotype distributions of patients with cancer of the breast and uterus at the Presbyterian Hospital, New York, and these distributions show the patients to be predominantly endomorphic mesomorphs. As this tendency also appears in patients suffering from other diseases at the same hospital, it may well be due to selection. Sheldon and others (1949) also published a somatotype distribution of 100 men with pulmonary tuberculosis, which showed no striking tendency towards a particular somatotype. He suggested that tuberculosis is decidedly not associated with ectomorphy, but stated that many of the tuberculosis patients could have been described as asthenic mesomorphs. On the other hand, Berry and Nash (1955) showed that, in comparison with controls, normal males who subsequently developed tuberculosis tended to have relatively long narrow chests and hearts, i.e. ectomorphic characteristics.

Palmer, Jablon, and Edwards (1957) also showed that resistance to the development of tuberculosis in already tuberculin-positive men was strongly related to build, being less in tall than in short men, and less in light than in heavy men. There may therefore be a tuberculosis "type", but there is little evidence so far of a cancer "type", or of these two being identical.

Lastly, it is of some interest to note that a study of cancer mortality alone has already suggested that the probability that an individual female will ultimately die of cancer is approaching an equilibrium value of about $0 \cdot 18$ (Haybittle, 1962). This would be entirely consistent with the data presented in the present paper, since, as shown in Fig. 1, mortality from tuberculosis is now becoming very small and may soon be negligible and, if the cancer ratio for females in Fig. 2 stops rising and levels out at $0 \cdot 18$, the constancy of the combined tuberculosis and cancer ratio will be maintained.

\section{Summary}

Mortality rates from cancer and tuberculosis have been calculated in the birth-standardized populations of England and Wales for the period 19211959. It is shown that the decrease in the probability that an individual will ultimately die from tuberculosis has been almost exactly compensated for by the increase in the probability that he or she will die from cancer, so that the combined probability has remained approximately constant in the region of $0 \cdot 18$. This finding extends the previous cohort analysis made by Cherry (1925). The possibility of some common genetical factor predisposing to both cancer and tuberculosis is briefly discussed.

I am greatly indebted to Professor J. S. Mitchell for his interest in this work and for a number of most helpful discussions. I should also like to thank Mrs. P. C. Williams for her technical assistance.

\section{REFERENCES}

Berry, W. T. C. and Nash, F. A. (1955). Tubercle, 36, 164. Campbell, A. H. (1961a). Brit. J. Cancer, 15, 10; (1961b). Aust. Ann. Med., 10, 129.

Cherry, T. (1925). Med. J. Aust., 1, 581.

Coghlan, T. A. (1903). "Intercolonial Medical Congress of Australasia, Trans. Sixth Session, Hobart, Tas- $\varnothing$ mania, 1902"', p. 63. Vail, Government Printer, Hobart

Cruickshank, D. B. (1939). Papworth Res. Bull., 2, 1 . Pendragon Press, Papworth.

General Register Office (1957). "Cancer Statistics for" England and Wales 1901-55", by A. McKenzie, with additional material by R. A. M. Case and J. T. Pearson. Studies on Medical and Population Subjects, No. 13. H.M.S.O., London.

Goodhart, C. B. (1959), Practitioner, 182, 578.

Haybittle, J. L. (1962). Brit. J. prev. soc. Med., 16, 93.

Huxley, J. (1958). "Biological Aspects of Cancer". Allen and Unwin, London.

Mitchell, J. S., and Simon-Reuss, I. (1952). Brit. J. Cancer, 6, 305, 317.

Palmer, C. E., Jablon, S., and Edwards, P. Q. (1957). Amer. Rev. Tuberc., 76, 517.

Paxon, T. G. (1956). Brit. J. Cancer. 10, 623.

Pearl, R. (1929), Amer. J. Hyg., 9, 97.

Sheldon, W. H., Hartl, E. M., and McDermott, E. (1949). "Varieties of Delinquent Youth". Harper, New York. 